\title{
Transmission of multidrug-resistant tuberculosis in Shimen community in Shanghai, China: a molecular epidemiology study
}

Zhiying Han ${ }^{1 *+}$, Jing $\mathrm{Li}^{2+}$, Guomei Sun ${ }^{1}$, Kaikan Gu${ }^{1}$, Yangyi Zhang ${ }^{2}$, Hui Yao ${ }^{3}$ and Yuan Jiang ${ }^{2 *}$

\begin{abstract}
Background: Multidrug-resistant tuberculosis (MDR-TB) has become a major public health problem in China, with mounting evidence suggesting that recent transmission accounts for the majority of MDR-TB. Here we aimed to reveal the transmission pattern of an MDR-TB outbreak in the Jing'an District of Shanghai between 2010 and 2015.
\end{abstract}

Methods: We used whole-genome sequencing (WGS) to conduct genomic clustering analysis along with field epidemiological investigation to determine the transmission pattern and drug resistance profile of a cluster with ten MDR-TB patients in combining field epidemiological investigation.

Results: The ten MDR-TB patients with genotypically clustered Beijing lineage strains lived in a densely populated, old alley with direct or indirect contact history. The analysis of genomic data showed that the genetic distances of the ten strains (excluding drug-resistant mutations) were 0-20 single nucleotide polymorphisms (SNPs), with an average distance of 9 SNPS, suggesting that the ten MDR-TB patients were infected and developed the onset of illness by the recent transmission of $M$. tuberculosis. The genetic analysis confirmed definite epidemiological links between the clustered cases.

Conclusions: The integration of the genotyping tool in routine tuberculosis surveillance can play a substantial role in the detection of MDR-TB transmission events. The leverage of genomic analysis in combination with the epidemiological investigation could further elucidate transmission patterns. Whole-genome sequencing could be integrated into intensive case-finding strategies to identify missed cases of MDR-TB and strengthen efforts to interrupt transmission.

Keywords: Transmission, Molecular epidemiology, Multidrug-resistant tuberculosis

\section{Introduction}

According to the global tuberculosis report [1], there are an estimated 465,000 rifampicin-resistant tuberculosis (RR-TB) patients in 2019 worldwide, of which about $78 \%$ were multidrug-resistant tuberculosis (MDR-TB). China

\footnotetext{
*Correspondence: zhiying.han728@gmail.com; jiangyuan@scdc.sh.cn ${ }^{\dagger}$ Zhiying Han and Jing Li contributed equally to this work

${ }^{1}$ Department of Tuberculosis Prevention and Control, Jing'an District Center for Disease Control and Prevention, Shanghai 200072, China

${ }^{2}$ Tuberculosis Laboratory, Shanghai Municipal Center for Disease Control and Prevention, Shanghai 200036, China

Full list of author information is available at the end of the article
}

has the second-highest burden of MDR-TB and has approximately 65,000 incident cases of RR-TB in 2019 in the Chinese mainland, accounts for $14 \%$ of RR-TB worldwide [1]. According to the TB Information Management System in Shanghai, 3395 TB cases were reported in 2019 with a reported incidence of $25 / 100,000$, with an overall drug-resistance rate of $11.7 \%$ and MDR-TB of $3.7 \%$. Understanding the transmission pattern of MDR-TB is of great importance to prevent and control TB.

Due to the remarkable variability in the timing of disease progression after infection, it was difficult to measure and track the transmission of TB. The application of 
genotyping techniques of Mycobacterium tuberculosis (M. tuberculosis) provides a useful tool for researchers to explore the transmission patterns of TB and the risk factors associated with transmission [2]. Previous methods used for $M$. tuberculosis molecular epidemiology that have provided useful insight to understand transmission and differentiate between endogenous onset and exogenous reinfection of drug-resistant strains include insertion sequence 6110-restriction fragment length polymorphism (IS6110), spoligotyping, and variable numbers of tandem repeats (VNTR) techniques [3, 4]. Over time, the resolution of the genotyping tool has been ultimately improved as the development of high-throughput whole-genome sequencing (WGS) in the studies of molecular epidemiology of TB transmission. The WGS can confirm the variation of genetic diversity among clustered strains to construct a relatively accurate transmission chain may provide insight into source cases and potential missing cases, an advantage compared to the traditional epidemiological investigation and genotyping methods. [5-8]

A recent study has found that MDR-TB cases in Shanghai were mostly attributed to direct transmission of drugresistant strains in Shanghai, and part of the clustered strains was found in the Jing'an District [8]. Previously, to study transmission patterns of TB in this district, we conducted a molecular epidemiology study with the clinical M. tuberculosis isolates from patients diagnosed in Jing'an Designated Hospital from 2010 to 2015. By using the "9+3" VNTR genotyping loci set [9], we identified genotypically clustered strains. The largest identified cluster included ten MDR-TB cases, of which all the patients were living in the same community and had symptom onset over ten years. Here, we performed combined whole-genome sequencing analysis and field epidemiological investigation of these ten MDR-TB patients, to further understand the transmission pattern and the cause of this MDR-TB cluster. The result of this study could provide evidence to inform more efficient MDR-TB control strategies in urban areas.

\section{Methods}

\section{Data source}

At Jing'an Designated Hospital from 2010-2015, one hundred seventy-three pulmonary TB patients were diagnosed, of which 92 cases were culture positive. From the Shanghai Municipal Center for Disease Control and Prevention (Shanghai $C D C$ ), we collected 80 of 92 effective strain samples in this district for genotyping analysis. All of the M. tuberculosis isolates were sent to the reference laboratory for drug susceptibility testing (DST) using the proportion method on Lowenstein-Jensen medium at the following concentrations: rifampicin (RIF) $40 \mu \mathrm{g} / \mathrm{mL}$, isoniazid (INH) $0.2 \mu \mathrm{g} / \mathrm{mL}$, ethambutol (EMB) $2.0 \mu \mathrm{g} /$ $\mathrm{mL}$, and streptomycin (SM) $4.0 \mu \mathrm{g} / \mathrm{mL}$. As described previously, we conducted VNTR genotyping for all strains using the $9+3$ loci set with hypervariable loci that were developed specifically for discriminating of $M$. tuberculosis strains in China and identified 65 genotypes, with 22 strains forming 7 clusters. $[9,10]$ The largest cluster of ten MDR-TB patients was selected for further analysis in this study.

\section{Whole-genome sequencing, clustering analysis, and transmission inference}

We performed whole-genome sequencing analysis on MDR-TB strains from all ten patients. The genomic DNAs were extracted following the cetyl trimethyl ammonium bromide (CTAB) method. [11] The reference laboratory and data center at the Shanghai CDC performed the DNA library preparation and whole-genome sequencing using Illumina Hiseq 2500 with an average coverage of 137 times with a range from 112 to 180 times. Analysis of the raw sequencing data was completed using the standard process pipeline according to previous studies. [12] High quality and trimmed sequencing reads were mapped to $\mathrm{H} 37 \mathrm{Rv}$ reference sequence (NC_000962.3) using Bowtie2 (v2.2.9). [13] We used Samtools (v1.6) together with VarScan (v2.3.9) to call the single-nucleotide polymorphisms (SNPs) and identify fixed genomic variants (frequency $\geq 75 \%$ ). [14][14]. The fixed SNPs, excluding those in the region of PE-PGRS and PE-PPE genes, and drug-resistance associated genes, were combined into a concatenated alignment. We then used this alignment to construct a maximum-likelihood phylogeny using MEGA (version 9.0) with a general timereversible (GTR) substitution model and 1,000 bootstraps (Additional file 1: Figure S1 and S2, appendix). We defined genomic clusters as those strains within a threshold of twelve or fewer SNPs [8]. We determined the sublineage and drug-resistant gene mutations based on the WGS data analysis by using an in-house Perl script and TB-Profiler (v2.8.14). We also used the SpoTyping (version 2.0) to generate the spoligotyping pattern from the sequenced data [16].

We used a Bayesian evolutionary analysis to infer a timed phylogeny by sampling trees with BEAST (version 1.8.4) by using the concatenated alignment and labeled the time tips of each strain using the diagnosed date. We used the GTR substitution model with a coalescent constant population size and a strict molecular clock rate. The input XML file was modified to specify the number of invariant sites. The model was run using a Markov Chain Monte Carlo (MCMC) chain length of $10,000,000$ with a $10 \%$ burn-in. A maximum clade credibility tree was generated using TreeAnnotator (v2.4.7) 
and visualized using FigTree (v1.4.2). This timed phylogeny tree was then used as input for the transmission tree inference using the R package called TransPhylo and followed the previously described methods. [17] We used a Gamma distribution with a shape parameter of 1.3 and a rate parameter of 0.3 for the generation time density, with consideration of the generation time of tuberculosis that allowed a rapid progression to active disease after infection, but also the possibility of a long latent period before active progression. [17]

\section{Epidemiological investigation}

Epidemiological investigations were used to identify the persons, places, and behaviors associated with the transmission of $M$. tuberculosis between TB patients in this cluster. A standard questionnaire was used to collect information about the individual's place of residence, close contacts, interpersonal relationships, frequently visited locations, and the situation of hospital visits as previously described for this cluster [8]. Epidemiological links were defined as follows: "confirmed epidemiological links" were recognized if two patients were close contacts/acquaintances or resided in the same location; "probable epidemiological links" were recognized if two patients resided in a neighborhood or frequently shared common spaces.

All interviews were performed retrospectively by trained interviewers after obtaining informed consent. If the patient was not able to participate in the interview themselves, close contact of the patient (e.g., spouse, children, parents) was interviewed. On-site investigation of TB patients was carried out according to national TB control guidelines.

\section{Results}

Demographic features and TB history

Among the ten MDR-TB patients included, eight were male. Six patients had no previous history of pulmonary TB. Five of ten were retired persons, and another five were unemployed. The mean age was 56 years, with a range from 35 to 75 . (Table 1). Six patients had no history of antituberculous therapy, of which four patients were seriously ill. Of the four remaining patients with a previous history of treatment, three had severe disease. Half of the ten MDR-TB patients died and three of them were attributed to TB disease. One was discontinued due to drug side effects, and two were still in the period of follow-up (interrupted treatment due to severe side effects). Only one patient was cured. (Table 1 ).

\section{Epidemiological investigation}

Among the ten patients, nine had confirmed epidemiological links, including two couples (patient ID: 20100183 and 2012-1261; 2012-0659 and 2012-1614) and one father-and son-pair (2010-1007 and 2016-0569). These nine patients were acquaintances: 8 resided in the same alley, the 9th patient (2012-1220) lived on the ground floor of another alley within $200 \mathrm{~m}$, and

Table 1 Characteristics of multidrug-resistant tuberculosis in the cluster $(n=10)$

\begin{tabular}{|c|c|c|c|c|c|c|c|}
\hline Patient ID & Sex & Age, years & $\begin{array}{l}\text { Previous } \\
\text { treatment }\end{array}$ & $\begin{array}{l}\text { Onset/diagnosed } \\
\text { time (DD-MM-YY) }\end{array}$ & Treatment outcomes & Relationship & Nature of epidemiological link \\
\hline $2010-0183$ & Male & 49 & No & $\begin{array}{l}\text { 10-Sep-09 } \\
\text { 15-Nov-09 }\end{array}$ & Died & Couple & \multirow{9}{*}{$\begin{array}{l}\text { All lived in the same residential } \\
\text { community or alley; Neighbor- } \\
\text { hood; } \\
\text { Game room or restaurant within } \\
\text { the alley }\end{array}$} \\
\hline $2012-1261$ & Female & 35 & No & $\begin{array}{l}\text { 11-May-12 } \\
\text { 23-May-12 }\end{array}$ & Still on treatment ${ }^{*}$ & & \\
\hline $2010-1007$ & Male & 75 & No & $\begin{array}{l}\text { 10-Jul-09 } \\
\text { 21-Oct-09 }\end{array}$ & Interrupted & Father and son & \\
\hline $2016-0569$ & Male & 58 & Yes & $\begin{array}{l}\text { 03-Jan-15 } \\
\text { 26-Jan-15 }\end{array}$ & Still on treatment* & & \\
\hline $2012-0659$ & Female & 52 & Yes & $\begin{array}{l}\text { 01-Mar-06 } \\
\text { 21-Jul-11 }\end{array}$ & Died & Couple & \\
\hline $2012-1614$ & Male & 58 & Yes & $\begin{array}{l}\text { 02-Apr-06 } \\
\text { 18-Sep-12 }\end{array}$ & Died & & \\
\hline $2013-0285$ & Male & 53 & No & $\begin{array}{l}\text { 25-Dec-12 } \\
\text { 10-Jan-13 }\end{array}$ & Cured & Neighborhood & \\
\hline $2012-1050$ & Male & 56 & No & $\begin{array}{l}\text { 01-Mar-12 } \\
\text { 13-Mar-12 }\end{array}$ & Died & & \\
\hline $2012-1220$ & Male & 63 & Yes & $\begin{array}{l}\text { 01-Mar-09 } \\
\text { 17-Jan-12 }\end{array}$ & Died & ND & \\
\hline 2010-1008 & Male & 61 & No & $\begin{array}{l}\text { 28-Mar-10 } \\
\text { 06-May-10 }\end{array}$ & Treatment completed & ND & Casually visit the game room \\
\hline
\end{tabular}

*Patients 2012-1261 and 2016-0569 were still on treatment when the investigation was performed 
owned a fast-food restaurant. The remaining patient had "probable epidemiological links" as defined above, and lived in an alley about two kilometers away from the address of 9 other patients (Table 1). Eight of the ten patients who visited the same game room frequently in this ally.

Table 2 shows the dates of symptom onset, TB diagnosis, treatment course, and hospitalization. Both patients 2012-0659 and 2012-1614, who belonged to a couple, experience symptom onset in 2006 . They experienced long delays and interrupted treatment from 2006 to 2011 . The remaining eight patients experienced a median diagnosis delay of 15 days of seeking health care (IQR, 12-65 days). Although ten patients visited the same hospital to follow up, only two of the ten patients had an overlapping hospital stay for a few days during the middle of their hospitalization. (Table 2).

\section{Sublineage, drug resistance mutations, and phylogenetic} analysis

All ten MDR-TB patients were infected with strains belonging to the Beijing family in sublineage 2.2.2 (Modern Beijing strain), and had the same spologotyping pattern (000000000003771). Table 3 showed the drug susceptibility profiles and drug resistance mutations in related genes. Each of the ten patients had the same fixed INH, RIF, and SM resistant related gene mutations (katG-315S/T, rpoB-450S/L, and rpsL-43 K/R respectively). The other DR mutations were described below in the transmission chain analysis. We did not observe any fixed drug-resistant mutations to second-line anti-TB drugs, including amikacin, kanamycin, and capreomycin.

WGS showed that the genomic differences between any two strains (excluding drug-resistant mutations) ranged from 0 to 20 SNPs, and the average difference was 9.1 SNPs (Table 4), consistent with the VNTR genotyping analysis and supporting recent transmission. We built a maximum-likelihood (M-L) phylogenetic tree based on

Table 2 The time (by year and month) of onset of symptoms, confirmation of diagnosis, treatment course and duration of hospitalization of the ten MDR-TB cases

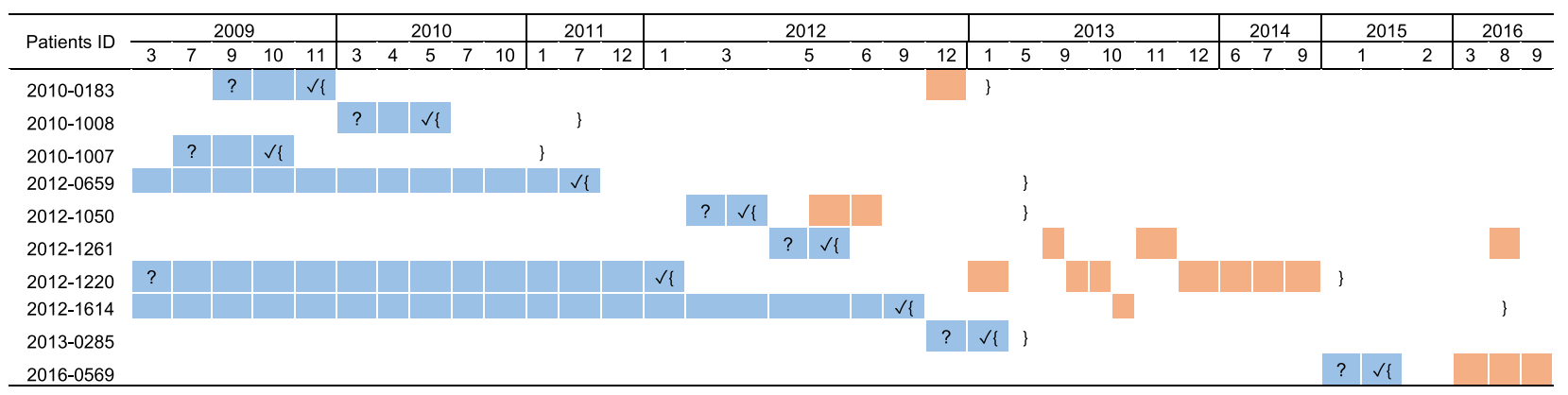

$?=$ onset of TB like symptoms (*Patient 2012-0659 and 2012-1614 had the TB symptom back to the time of 2003); $\checkmark=$ confirmation of diagnosis; \{\} represents the treatment course, patients 2016-0569 and 2012-1261 were still on treatment; the cells in blue represent the delay of diagnosis and the cells in orange represents hospitalization

Table 3 Profile of phenotypic resistance and drug-resistant related gene mutants of the MDR-TB cases

\begin{tabular}{|c|c|c|c|c|c|c|c|c|c|c|}
\hline Patient ID & $\begin{array}{l}\text { Phenotypic } \\
\text { resistance } \\
\text { (SIRE) }\end{array}$ & INH & RIF & SM & EMB & PZA & FLQ & Am & $\mathrm{Cm}$ & $\mathrm{Km}$ \\
\hline 2010-1007 & $R-R-R-R$ & katG-S315T & $r p o B-S 450 \mathrm{~L}$ & rpsL-K43R & embB-M306V & pncA-V155G & gyrA-A90V & - & - & - \\
\hline 2010-1008 & $R-R-R-R$ & katG-S315T & rpoB-S450L & rpsL-K43R & embB-M306V & pncA-V155G & gyrA-A90V & - & - & - \\
\hline 2010-0183 & $R-R-R-R$ & katG-S315T & rpoB-S450L & rpsL-K43R & embB-M306V & pncA-V155G & - & - & - & - \\
\hline 2012-1050 & $R-R-R-R$ & katG-S315T & rpoB-S450L & $\operatorname{rpsL-K43R}$ & embB-M306V & pncA-V155G & - & - & - & - \\
\hline $2012-1220$ & $R-R-R-R$ & katG-S315T & rpoB-S450L & rpsL-K43R & embB-M306V & pncA-V155G & gyrA-D94G & - & - & - \\
\hline $2012-1261$ & R-R-R-R & katG-S315T & rpoB-S450L & rpsL-K43R & embB-M306V & pncA-T47P & - & - & - & - \\
\hline 2012-0659 & $R-R-R-R$ & katG-S315T & rpoB-S450L & rpsL-K43R & embB-M306V & pncA-C14Y & gyrA-D94A & - & - & - \\
\hline 2013-0285 & R-R-R- R & katG-S315T & rpoB-S450L & rpsL-K43R & embB-M306V & pncA-V155G & - & - & - & - \\
\hline 2016-0569 & R-R-R-S & katG-S315T & rpoB-S450L & rpsL-K43R & - & - & - & - & - & - \\
\hline 2012-1614 & R-R-R-S & katG-S315T & rpoB-S450L & rpsL-K43R & - & - & - & - & - & - \\
\hline
\end{tabular}

$R$ resistant, $S$ susceptible, INH isoniazid, $R I F$ rifampicin, EMB ethambutol, $P Z A$ pyrazinamide, $S M$ streptomyces $F L Q$ fluoroquinolones, $A m$ amikacin, $K m$ kanamycin, $C m$ capreomycin 
Table 4 Pairwise SNPs distance between the 10 MDR-TB strains in the cluster

\begin{tabular}{|c|c|c|c|c|c|c|c|c|c|c|}
\hline SNP matrix & $\begin{array}{l}\text { o } \\
\text { ㅇ } \\
\text { b } \\
-1 \\
0\end{array}$ & 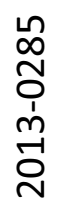 & 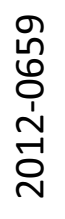 & 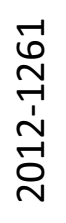 & 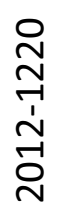 & 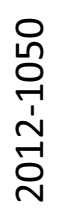 & $\begin{array}{l}m \\
\infty \\
-1 \\
0 \\
0 \\
0 \\
-1 \\
\text { ․ }\end{array}$ & 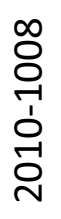 & 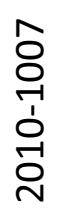 & 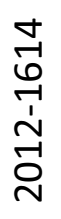 \\
\hline 2016-0569 & 0 & 13 & 11 & 11 & 13 & 12 & 11 & 13 & 20 & 7 \\
\hline 2013-0285 & 13 & 0 & 8 & 10 & 8 & 5 & 6 & 10 & 16 & 6 \\
\hline 2012-0659 & 11 & 8 & 0 & 8 & 7 & 7 & 6 & 10 & 17 & 4 \\
\hline $2012-1261$ & 11 & 10 & 8 & 0 & 10 & 9 & 8 & 13 & 18 & 4 \\
\hline $2012-1220$ & 13 & 8 & 7 & 10 & 0 & 7 & 2 & 7 & 17 & 6 \\
\hline 2012-1050 & 12 & 5 & 7 & 9 & 7 & 0 & 5 & 9 & 15 & 5 \\
\hline 2010-0183 & 11 & 6 & 6 & 8 & 2 & 5 & 0 & 7 & 15 & 4 \\
\hline 2010-1008 & 13 & 10 & 10 & 13 & 7 & 9 & 7 & 0 & 1 & 9 \\
\hline 2010-1007 & 20 & 16 & 17 & 18 & 17 & 15 & 15 & 1 & 0 & 14 \\
\hline 2012-1614 & 7 & 6 & 4 & 4 & 6 & 5 & 4 & 9 & 14 & 0 \\
\hline
\end{tabular}

the SNPs alignment (Fig. 1) and mapped the drug resistance mutations to the $\mathrm{M}-\mathrm{L}$ tree. The accumulation of drug-resistant mutations, along with the drug-susceptibility testing, suggest that this cluster arose from the transmission of MDR M. tuberculosis strain.

\section{Transmission chain inference}

Additiona file 1: Figure S3 shows the transmission inference based on the timed phylogeny tree analysis. The putative transmission source or index case was initialed before 2006, which was in consistent with the investigation that patients 2012-1614 and 2012-0659, who were a couple, had presented symptoms in March and April 2006, respectively. Both strains from this couple were in the root of the M-L tree in Fig. 1, indicating the putative early transmission events related to these two patients.

Based on the alignment of the SNPs of the ten MDR $M$. tuberculosis strains, we mapped the drug-resistant mutations on the phylogenetic tree of each node (Fig. 1). We observed consistent mutation profiles of drug resistance genes of INH, RFP, and SM for each of the ten strains. Meanwhile, those strains located at the end branch had several unique drug-resistance mutations, including the gyrA A90C in 2010-1007 and 2010-1008, and gyrA D94G in 2012-1220. These findings provide further evidence of direct transmission of MDR-TB strains and suggest the acquisition of additional drug-resistance mutation during the transmission of MDR-TB strains.

The timed-tree in Additional file 1: Figure S3 also suggests at least two transmission routes initialed by the source case, one subsequentially transmitted to the four individuals, including 2012-1261, 2016-0569, and the patients in a couple 2012-1614 and 2012-0659. This finding was supported by the relatively small genetic differences (less than four SNPs) between the patients 2012-1614 (husband of patient 2012-0659), 2012-1261 (living in the same alley), and 2010-0183 (living in the same alley) (Table 4). Another route includes several transmission events to the remaining six cases. The shared mutation of both ethambutol (EMB) and pyrazinamide (PZA) genes among these six patients (Table 3) suggest that they may be transmitted from a common source case.

\section{Discussion}

Based on a genotyping analysis of $M$. tuberculosis in a district in Shanghai, we conducted a genomic and epidemiological analysis of ten MDR-TB strain cluster and described the putative transmission chain of this cluster. With the development of next-generation high-throughput sequencing technology, whole-genome sequencing analysis has been gradually applied to the molecular 

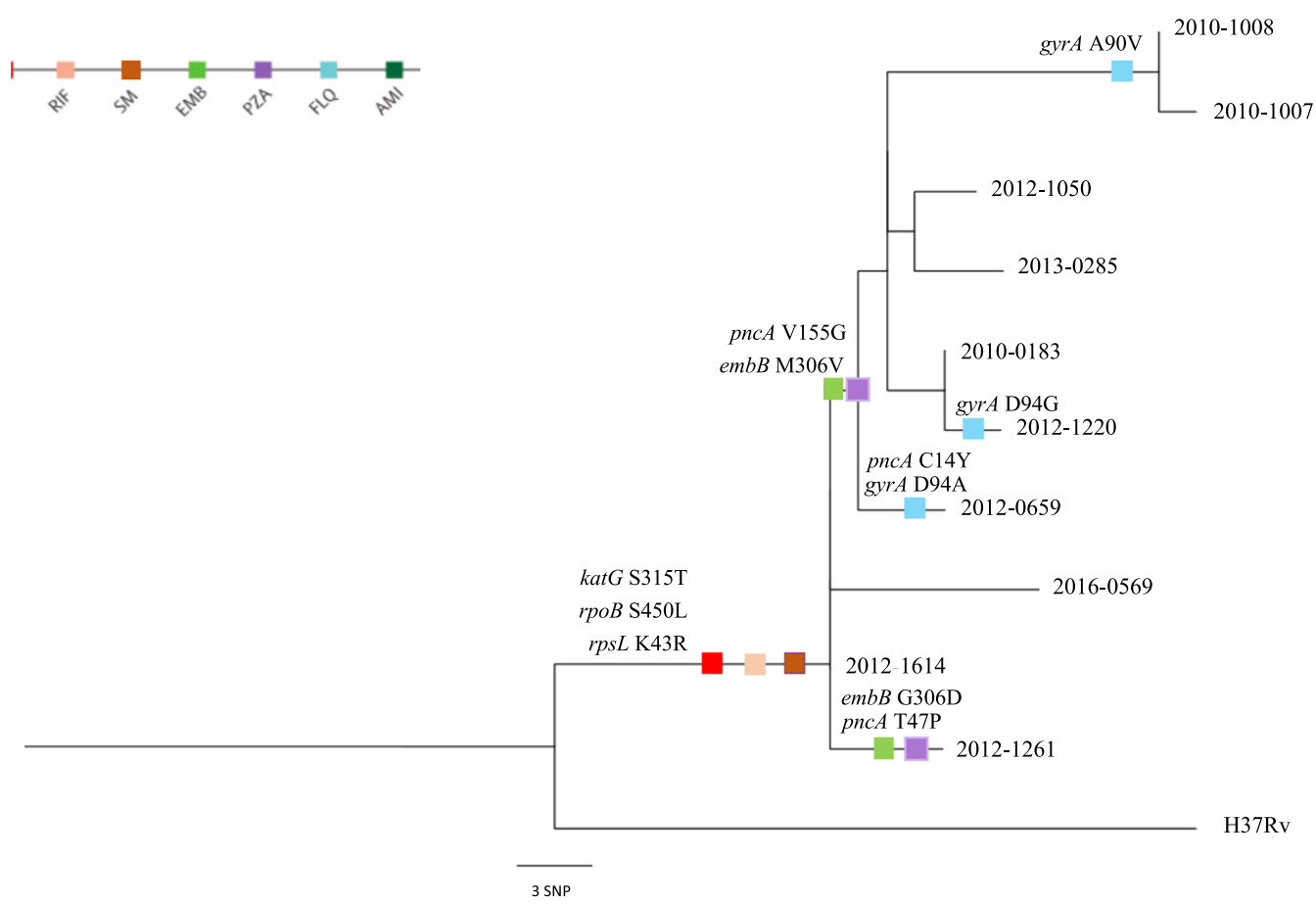

Fig. 1 Maximum-likelihood, single-nucleotide polymorphism-based phylogeny of multidrug-resistant isolates and mutations of drug-resistant genes among ten clustered multidrug-resistant TB strains. This phylogenetic tree was based on 10 sequences with a total 68 core single nucleotide variant positions (MEGA v9.0)

epidemiological studies of M. tuberculosis [7, 18, 19], which can determine the variation and transmission source in a transmission chain [20]. Because the reverse mutation of single nucleotide polymorphism (SNP) is rare $[21,22]$, WGS data can accurately describe transmission direction by comparing differences in SNPs between TB strains in a cluster.

We combined WGS and field epidemiological investigations from a cluster of ten MDR-TB patients to infer the transmission route. Current evidence and the transmission tree (Figs. 1 and 2) suggest that this cluster initialed as early as 2006, with unsampled source cases. The source case, likely infected before 2006, transmitted the MDR M. tuberculosis strain to at least 9 cases in this community. The timed tree (Additional file 1: Figure S3) indicated an unsampled source case for this cluster. Due to the lack of previous strains from these two patients, we cannot rule out the possibility of other source cases. The timed-tree also showed that there were multiple transmission events in this community; however, due to the relocation of residents living in this alley, the transmission of this MDR-TB strain may seed in other communities, and further contact tracing should be reinforced.

In the field epidemiological investigation, we found that these transmission events likely occurred in the household or a commonly frequented game room.
There were reports in China that the transmission of TB may be due to accidental exposure [23], which indicated that most patients in a cluster had no apparent history of contact with other patients. Many molecular epidemiological studies abroad reported a lower proportion of cluster cases who had determined contact history [24-26]. Our investigation found that some of the patients in this cluster had occasional contacts in the game room, suggesting that short contacts that do not typically qualify as long-term contacts may lead to infection. In this study, we were impressed that this neighborhood was one of the largest traditional residences in Shanghai, which was eventually demolished in April 2013. Before the demolition, there were 2500 households and about 4000 residents living in the area of 40,000 square meters, and the population density was $1 / 10 \mathrm{~m}^{2}$. There were several commonly frequented, poorly ventilated fast-food restaurants and family game rooms.

As a result of the relocation and other historical reasons, many of the tenants of the alley, who may have been exposed moved away. Given the suggestion that casual contacts may lead to successful transmission, public health officials should take measures to discover and treat MDR-TB patients among those potentially exposed households to control the transmission of MDR-TB. This 


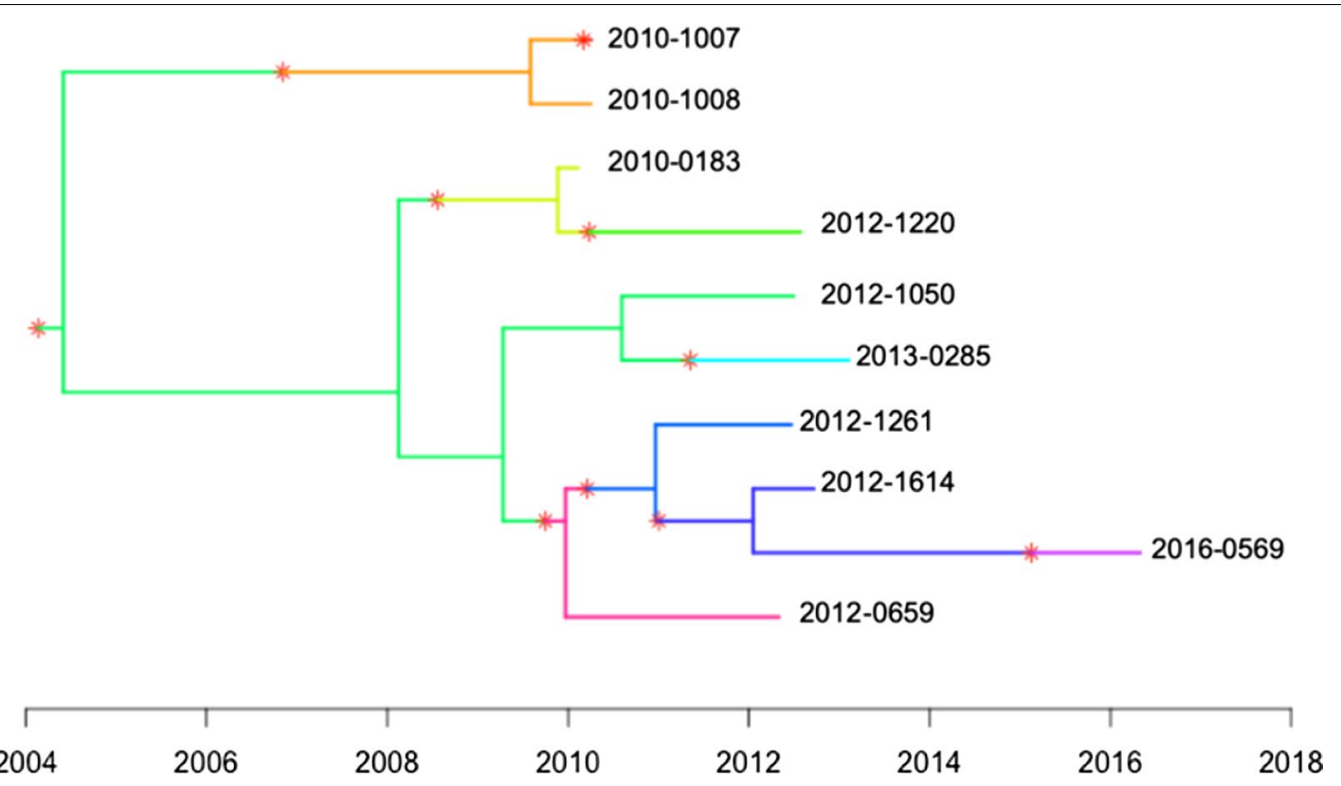

Fig. 2 Transmission tree based on the time-labeled phylogenic tree of the ten MDR-TB patients. The star and the change of color represent the occurrence of transmission event or new infection

also raises the limitation that this demolition of the alley could lead to an incomplete contact evaluation.

MDR-TB patients may have a longer extended period of infection due to delayed diagnosis and treatment, and delays in sputum Mycobacteria conversion. [27, 28]. The treatment of MDR-TB in China has a relatively low cure or completion rate $(<50 \%)$ [28]. In this study of $10 \mathrm{MDR}$ TB patients, only one patient was cured. Incomplete treatment may facilitate the transmission of MDR-TB. Our findings suggest that a surveillance system integrating molecular genotyping may detect putative transmission of MDR-TB strains in the community and inform the epidemiological investigation of the transmission network. Routine or ideally real-time implementation of the genotyping and WGS tools may be informative to initial the alarm of possible outbreaks. However, due to the technological and financial reasons, the powerful genomic sequencing is not available or at impractical costs in many high TB burden regions including the rural parts of China. Therefore, the WGS cannot be presented as the only solution capable of tracing the epidemiological relationships for the control of tuberculosis in different countries, as it has been systematically emphasized in scientific publications on the molecular characterization of M. tuberculosis.

In addition to the transmission of drug-resistant strains, we demonstrate evidence of additional acquisition of drug-resistant mutations. WGS data analysis demonstrated that during the course of transmission, INH, SM, and RIF related resistance mutations were fixed, and EMB, PZA, and FLQ resistance mutations were accumulated gradually in several MDR-TB cases located in the end-branch of the phylogeny tree. The accumulation of additional drug-resistant mutations may result from incomplete or interrupted treatment due to serious treatment adverse effects and/or low treatment success rates. Treatment programs for MDR-TB patients should be individualized and standardized based on (DST) to increase the probability of successful treatment shorten the infectious period. Zhang and colleagues [29] found that adverse factors affecting the treatment effect of MDR-TB patients included long-term treatment and the initial drug-resistant types, suggesting short-term and effective treatment strategies may be crucial to contain the spreading of MDR-TB.

\section{Conclusions}

In summary, we observed a local transmission of MDR-TB in one relocated-community in Jing'an district in Shanghai. Our findings suggest that intensive TB screening might help to control local TB epidemics, especially in settings where there is a local transmission of MDR-TB. In order to impede transmission, public health efforts should increase the intensity of the active case finding of MDR-TB and target interventions to shared spaces that are commonly frequented, even for a short amount of time. This is especially true of poorly ventilated spaces. Additionally, rapid diagnostic technology to screen for TB and drug-resistance 
may enhance the early detection of MDR-TB. These intensive interventions could improve the monitoring, follow-up, and management of drug resistance TB patients, and may ultimately reduce the transmission of MDR-TB.

\section{Abbreviations}

MDR-TB: Multidrug-resistant TB; WGS: Whole-genome sequencing; SNPs: Single nucleotide polymorphisms; VNTR: Variable numbers of tandem repeats; RIF: Rifampicin; INH: Isoniazid; EMB: Ethambutol; SM: Streptomycin; EMB: Ethambutol; PZA: Pyrazinamide; CTAB: Cetyl trimethyl ammonium bromide; MCMC: Markov Chain Monte Carlo.

\section{Supplementary Information}

The online version contains supplementary material available at https://doi. org/10.1186/s12879-021-06725-0.

Additional file 1: Figure S1. Maximum-likelihood tree of ten MDR-TB strains and H37Rv. The bootstrap was showed as percentage of 1000 runs. Figure S2. Maximum-likelihood tree of ten MDR-TB strains. The bootstrap was showed as percentage of 1000 runs. Figure S3. Estimated transmission tree based on the time-labeled phylogenic tree of the ten MDR-TB patients. The star and the change of color represent the occurrence of transmission event or new infection.

\section{Acknowledgements}

We thank the Shanghai CDC TB Laboratory staff for providing the TB isolates for this study. We thank the Jing'an District Center for Disease Control and Prevention and the Second Shimen Road Community Health Center for their assistance with an epidemiological investigation.

\section{Authors' contributions}

$\mathrm{ZH}, \mathrm{YJ}$, and $\mathrm{J}$ contributed to the conception, design, and management of the study. GS, KG, and HY contributed to the questionnaire design. JL and $Y Z$ contributed to the demographic and epidemiological data collection. ZH, GS, $K G$, and $H Y$ contributed to the epidemiological investigations. $Y Z, J L$, and $Y J$ contributed to laboratory testing and molecular data analysis. $Z \mathrm{H}, \mathrm{YJ}$, and $\mathrm{J}$ prepared and reviewed the manuscript. All authors read and approved the final manuscript.

\section{Funding}

This work is supported by Public Health research Project of Science and Technology Committee of Jing'an District, Shanghai (No. 2020GW01) and National Key Research and Development Program of China (No. 2017YFD0500301).

\section{Availability of data and materials}

All data generated or analyzed during this study are included in this published article and its Additional file 1. All the sorted BAM files of the sequences were deposited in the NCBI Bioproject (PRJNA679962).

\section{Declarations}

\section{Ethics approval and consent to participate}

The study protocol was approved by the Ethical Review Committee at the Shanghai CDC (2014-1). All the participants in this study provided informed consent before they were interviewed in their household or the community health center/clinics. An investigation team including at least one local community health worker and one CDC staff conducted the investigation. All investigations and methods were carried out in accordance with Shanghai CDC guidelines and regulations.

\section{Consent for publication}

Not applicable.

\section{Competing interests}

The authors declare that they have no competing interests.

\section{Author details}

${ }^{1}$ Department of Tuberculosis Prevention and Control, Jing'an District Center for Disease Control and Prevention, Shanghai 200072, China. ${ }^{2}$ Tuberculosis Laboratory, Shanghai Municipal Center for Disease Control and Prevention, Shanghai 200036, China. ${ }^{3}$ Second Shimen Road Community Health Center, Shanghai, China.

Received: 8 November 2020 Accepted: 20 August 2021

Published online: 29 October 2021

\section{References}

1. WHO: Global Tuberculosis report 2020. 2020. http://www.who.int/tb/ publications/global_report/en/2020. Accessed Mar 182021

2. Gardy JL, Johnston JC, Ho Sui SJ, Cook VJ, Shah L, Brodkin E, Rempel S, Moore R, Zhao Y, Holt R, et al. Whole-genome sequencing and social-network analysis of a tuberculosis outbreak. N Engl J Med. 2011;364(8):730-9.

3. Allix-Beguec C, Fauville-Dufaux M, Supply P. Three-year population-based evaluation of standardized mycobacterial interspersed repetitive-unitvariable-number tandem-repeat typing of Mycobacterium tuberculosis. J Clin Microbiol. 2008;46(4):1398-406.

4. Barnes PF, Cave MD. Molecular epidemiology of tuberculosis. N Engl J Med. 2003;349(12):1149-56.

5. Guthrie JL, Delli Pizzi A, Roth D, Kong C, Jorgensen D, Rodrigues M, Tang P, Cook VJ, Johnston J, Gardy JL. Genotyping and whole-genome sequencing to identify tuberculosis transmission to pediatric patients in British Columbia, Canada, 2005-2014. J Infect Dis. 2018;218(7):1155-63.

6. Guthrie JL, Strudwick L, Roberts B, Allen M, McFadzen J, Roth D, Jorgensen $D$, Rodrigues $M$, Tang $P$, Hanley $B$, et al. Whole genome sequencing for improved understanding of Mycobacterium tuberculosis transmission in a remote circumpolar region. Epidemiol Infect. 2019;147:e188.

7. Luo T, Yang C, Peng Y, Lu L, Sun G, Wu J, Jin X, Hong J, Li F, Mei J, et al. Whole-genome sequencing to detect recent transmission of Mycobacterium tuberculosis in settings with a high burden of tuberculosis. Tuberculosis (Edinb). 2014;94(4):434-40.

8. Yang C, Luo T, Shen X, Wu J, Gan M, Xu P, Wu Z, Lin S, Tian J, Liu Q, et al. Transmission of multidrug-resistant Mycobacterium tuberculosis in Shanghai, China: a retrospective observational study using wholegenome sequencing and epidemiological investigation. Lancet Infect Dis. 2017;17(3):275-84.

9. Luo T, Yang C, Pang Y, Zhao Y, Mei J, Gao Q. Development of a hierarchical variable-number tandem repeat typing scheme for Mycobacterium tuberculosis in China. PLoS ONE. 2014;9(2):e89726.

10. Han ZY, Li J, Gu KK, Sun GM, Jiang Y, Zhang YY, Xu B. Recent transmission of pulmonary tuberculosis and its influencing factors in Jing'an district, Shanghai, 2010-2015. Zhonghua Liu Xing Bing Xue Za Zhi. 2018;39(10):1339-45.

11. Larsen MH, Biermann K, Tandberg S, Hsu T, Jacobs WR Jr. Genetic Manipulation of Mycobacterium tuberculosis. Curr Protoc Microbiol. 2007. https:// doi.org/10.1002/9780471729259.mc10a02s6.

12. Joshi N, Fass J: Sickle: a sliding-window, adaptive, quality-based trimming tool for FastQ files. (Version 1.33) [Software]. 2011

13. Langmead B, Salzberg SL. Fast gapped-read alignment with Bowtie 2. Nat Methods. 2012;9(4):357-9.

14. Li H, Handsaker B, Wysoker A, Fennell T, Ruan J, Homer N, Marth G, Abecasis $G$, Durbin R. Genome project data processing $S$ : the sequence alignment/map format and SAMtools. Bioinformatics. 2009;25(16):2078-9.

15. Koboldt DC, Zhang Q, Larson DE, Shen D, McLellan MD, Lin L, Miller CA, Mardis ER, Ding L, Wilson RK. VarScan 2: somatic mutation and copy number alteration discovery in cancer by exome sequencing. Genome Res. 2012;22(3):568-76.

16. Xia E, Teo YY, Ong RT. SpoTyping: fast and accurate in silico Mycobacterium spoligotyping from sequence reads. Genome Med. 2016;8(1):19.

17. Didelot X, Fraser C, Gardy J, Colijn C. Genomic infectious disease epidemiology in partially sampled and ongoing outbreaks. Mol Biol Evol. 2017:34(4):997-1007. 
18. Kohl TA, Diel R, Harmsen D, Rothganger J, Walter KM, Merker M, Weniger T, Niemann S. Whole-genome-based Mycobacterium tuberculosis surveillance: a standardized, portable, and expandable approach. J Clin Microbiol. 2014;52(7):2479-86.

19. Roetzer A, Diel R, Kohl TA, Ruckert C, Nubel U, Blom J, Wirth T, Jaenicke S, Schuback S, Rusch-Gerdes S, et al. Whole genome sequencing versus traditional genotyping for investigation of a Mycobacterium tuberculosis outbreak: a longitudinal molecular epidemiological study. PLoS Med. 2013:10(2):e1001387.

20. Walker TM, Monk P, Smith EG, Peto TE. Contact investigations for outbreaks of Mycobacterium tuberculosis: advances through whole genome sequencing. Clin Microbiol Infect. 2013;19(9):796-802.

21. Comas I, Coscolla M, Luo T, Borrell S, Holt KE, Kato-Maeda M, Parkhill J, Malla B, Berg S, Thwaites G, et al. Out-of-Africa migration and Neolithic coexpansion of Mycobacterium tuberculosis with modern humans. Nat Genet. 2013;45(10):1176-U1311.

22. Casali N, Nikolayevskyy V, Balabanova Y, Ignatyeva O, Kontsevaya I, Harris SR, Bentley SD, Parkhill J, Nejentsev S, Hoffner SE, et al. Microevolution of extensively drug-resistant tuberculosis in Russia. Genome Res. 2012;22(4):735-45.

23. $X u$ B, Hu Y, Wang WB, Jiang WL. Molecular-epidemiological study on the transmission of drug resistant tuberculosis and its influencing factors in rural areas of eastern China. Zhonghua Liu Xing Bing Xue Za Zhi. 2010;31(5):525-9.

24. Bishai WR, Graham NM, Harrington S, Pope DS, Hooper N, Astemborski J, Sheely L, Vlahov D, Glass GE, Chaisson RE. Molecular and geographic patterns of tuberculosis transmission after 15 years of directly observed therapy. JAMA. 1998;280(19):1679-84.

25. Small PM, Hopewell PC, Singh SP, Paz A, Parsonnet J, Ruston DC, Schecter GF, Daley CL, Schoolnik GK. The epidemiology of tuberculosis in San Francisco. A population-based study using conventional and molecular methods. N Engl J Med. 1994;330(24):1703-9.

26. Valway SE, Sanchez MP, Shinnick TF, Orme I, Agerton T, Hoy D, Jones JS, Westmoreland H, Onorato IM. An outbreak involving extensive transmission of a virulent strain of Mycobacterium tuberculosis. N Engl J Med. 1998;338(10):633-9.

27. He GX, Wang HY, Borgdorff MW, van Soolingen D, van der Werf MJ, Liu ZM, Li XZ, Guo H, Zhao YL, Varma JK, et al. Multidrug-resistant tuberculosis, People's Republic of China, 2007-2009. Emerg Infect Dis. 2011;17(10):1831-8.

28. Zhao YL, Xu SF, Wang LX, Chin DP, Wang SF, Jiang GL, Xia H, Zhou Y, Li Q, Ou XC, et al. National survey of drug-resistant tuberculosis in China. New Engl J Med. 2012;366(23):2161-70.

29. Zhang Q, Wu ZY, Zhang ZR, Sha W, Shen X, Xiao HP. Efficacy and effect of free treatment on multidrug-resistant tuberculosis. Exp Ther Med. 2016;11(3):777-82.

\section{Publisher's Note}

Springer Nature remains neutral with regard to jurisdictional claims in published maps and institutional affiliations.
Ready to submit your research? Choose BMC and benefit from:

- fast, convenient online submission

- thorough peer review by experienced researchers in your field

- rapid publication on acceptance

- support for research data, including large and complex data types

- gold Open Access which fosters wider collaboration and increased citations

- maximum visibility for your research: over 100M website views per year

At BMC, research is always in progress.

Learn more biomedcentral.com/submissions 